Pirle Simulations of Second-Order Fermi Acceleration of Electrons:

Solving the Injection Problem

authoris, Galen R. Gisler

submirted to Proceedings of Particle Acceleration in Cosmic Plasmas Cunference. Newark, DE, 2-4 December 1991

\title{
DISX'LAIMFR
}

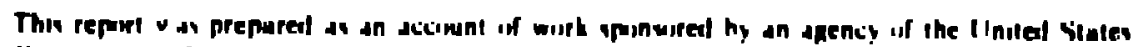
Cinvernment Nether the I'nited Sidtes fiuvernment now ans agenct thereuf. nut an! uf their

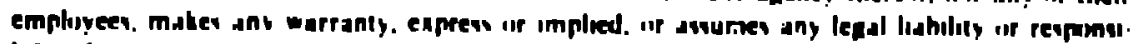

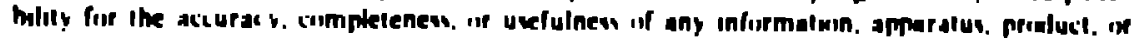

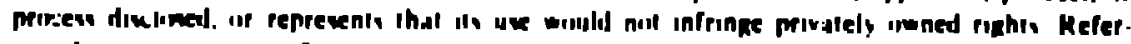

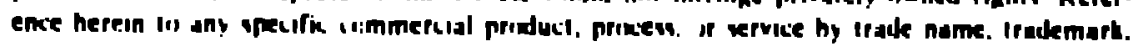

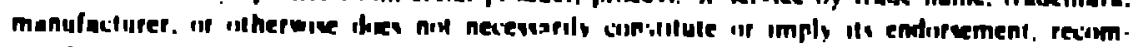

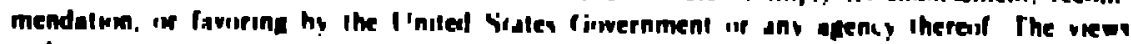

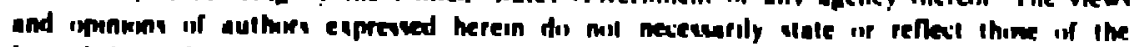
I'nired Siales finwernment uf any anency Iherest
\end{abstract}

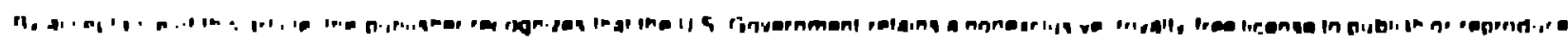

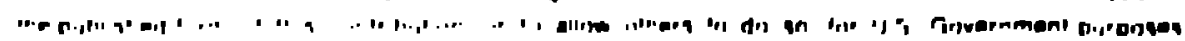

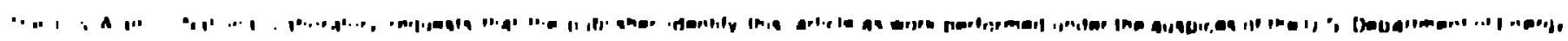

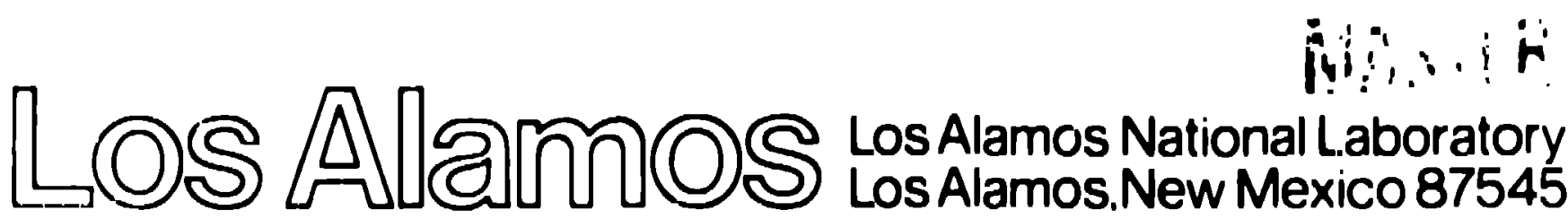




\title{
Simulations of Second-Order Fermi Acceleration of Electrons: Solving the Injection Problem
}

\author{
Gair:s Gisler \\ Spac : Plasma Physics. Los Alamor Narional Luboratom. \\ Lus Alamos. NM 87545.
}

\begin{abstract}
A estract
The boosting of electrons from a Maxwellian distributuon into a suprathermal power-law tail has long been recognized as an important bottleneck governing the subseyuent acceleration of some of these electrons to relativistic energies. This is the seed or injection problem. I study this boosting process using a test-particle simulation code. following the full equations of motion of tens of thousands of electrons chosen from a thermal populauion as they move through general time-dependent magnetic fields. Inhomogeneities in the magnetic field are provided by finite swarms of moving current loops with Maxwellian velocity distributions and power-law distributuons of loon size and dipole moment strength.

Whether bulk heating or boosting occurs is found wo depeild on the size c:t the swarm thermal speed compared ro the electuon thermal speed. When the swarm thermal speed is compirable to the electron thermal speed the entire electron population is heated by encouniers with the rapidly moving current loops. approxime'ely preserving the Maxwellian character of the electron distribution. On the other hand. at very low swarm thermal speeds there is no bulk heaung: instead one percent or fewer of the electrons are boosed into a power-law supratiermal tail with a differential energy spectral index between -1 and 2 . Individual boosts of 2010 and more have been observed in samples of $50.0(1)$ electrons. Most of the strongly hoosted electrons have initial energies that are well helow the peak of the initial Miaxwellian.
\end{abstract}

\section{IVTROIX (TIOS)}

Stoxhastic acceleration. or second-order fiermı acceleration, is usually treated using a diffusion or Fokker-Planch approach" The production of a power-liw output energy spectrum from a monoxenerpetic input is readily accomplished. and there are well-known expressions lor the acceleration efficiency and for the diffusion covetficients.

In this paper I am interested in the more general probiem of what stochastic acceleration dixes to a Maxwellian input energy spectrum. This is related (t) the famous "sced" problem: l.uw dores the process of panticle energization get staned"? Arguably. mosst of the diffuse matter in the universe has an energy specturn th 1 is risughly Maxwellian. and the extraction of energetic particles from that therma matter is a core parn of the problem of the production of cosmic rays. A diffusion ap oroxinattion is not well suted (1) this extraction problem. The second law of themod, namies demands that the production of a suprathemul tail he an inefficient proxess. selecting a very few particles for special ereatment. Any proxess that ac's on the majority of particles can only transform one Maxwellian into another. that is, hy hulk hesting or cooling.

Related in this consideration is the fact that a thermal population oll partic les in contact with a population of scatterers will tend toward thermodynamic equilib. rium with the scatterers. (Dily if the configuration os limited in ypace or time. allowing

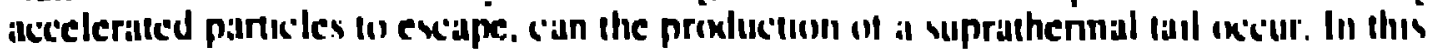


تlse the cunditions favoring equilibrium ure violated: the very lew energized purticles jepart fron, the system carrying their excess energy with them This turms out to be a very effective way for a plasma to get rid of excess riree energy.

The application to thermal electrons is complicated by the fict that these particles tynically have fast timesciales and small gyroradii. The electromagnetic field in the interstellar and interplanetary medium generally viries over much larger tume and spatial seales. and thermal electrons ure generally thought to respond "only" udiabatucally to the relatively small chinges on the electron siales.

It is wonh noting. however. that adiabatic changes can be sufficient for acceleration, if there is a way to "loxk in" the guins made during the favorable phases of the adiabatic cycles. An example of this is acceleration in a converging magnetic trap". This in essentially a tirst-order Fermi prosess in which an elecuron is rapped on a flux tube between two approathing magnetic mirrors. The electron gains energy on ench encounter with one of the mirrors until it has gained enough parallel momentum to enter the loss cone of one of the mirrons. As it escapes through the loss cone the electron loses sume energy, ruughly the energy gained in its list approach to the mimor. but previous adiabatic gains are preserved. During each encounter with an approaching mirror. the electron is accelerated hy the inductive electnc field produced by the motion of the mirror.

\section{ThE sivications}

I chosese to address this problem using the techniques of numerical simulation. A thermal population of particles is dropped unto a distribution of randomly moving magnetic inhomogeneities. and its evolution is observed. Because the extraction process is posited to be inerficient. I have done ic.sr-purlirle simulatıons rather than fully self-consistent ones. There are iwo reasons for this choice. one physical. the other numeric .l. Physically, since the proxess is ineificient, the fiew particles that are exuracted into the suprathermal tuil dor not greatly influence the evolution of the field inhomogeneities. Numerically. for crery extracied particle one needs to compute thousands of partecles that remain in the core of the distribution. and this makes velf-consistent culculations expensive and impracticul.

An example of this approach is piven in (iisler and I demons'. where we presunted simulations of electron acceleratuon in a closed flux ube with converging

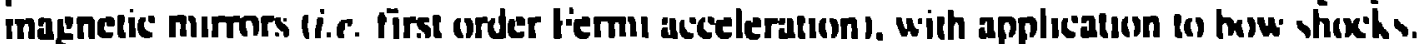
In tie present work I use a substantially upgraded version of that earlier conds.

'The magnetic suattering centers are represcnted as moving magnetic dipoles. The pirticles i which may he either electrons or ions) are followed in three dimensions along their orhits in the mngnetic fields of these dipoles. using an aduptive RungeKunta wheme which amply resolven the particle gyrofreyuency. The ciode is lully relativistic: "There is no) grid: the field sources all contrnhute for the field at any pinticle position. A puricle - Jamileonate upproach is used for the equations of motion. sol th: acteleration of a particle is a result of the lex-al inductive electric field at the particli: position from the motion of nearby dipoles.

The conde is implemented in TIIINK lissul on the Apple Mexinush computer. Menus allow ron-time adjustiment of all important phyvies parameters in the conde. almil provide control of the rest-time ploteng and orher cuupuss fiom the code.

Parricles are chosen at random firon a 3 .dimensional thermal mopulation. and Jropped into a sw unn of moving megnefic dipoles. In this paper. the dipole sw anm also has a 3 dimenvoinal Maxwellian velexity dosinhution. and that distnhumons in

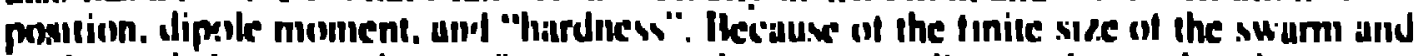

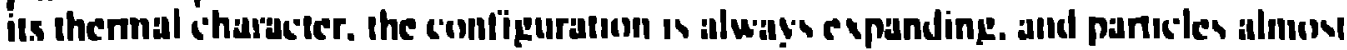


always manage to exwape. There are tuenty dipoles in the swarm. and to avold any statistical peculiarities caused by this smin'l number. a new' swam from the sume population is chosen after every hundred particles have been run. The dipoles are unaccelerated. that is. they are intinitely massive compared (s) the particles. Sume individual sw'arms have been lisund to be much more effective than the average at producing suprathermis particles. and we have used these to try to understand how particles are selected for atceleration.

Since the full eyuation of motion is uxed. parnicles will scatter off the magnetic dipoles or not. acecording to the appropnate physics. Particle trapping c'an also (xccur. as follows. Two moving dipoles with opposing tields approach each other $s$ ) that the field between them is iemporarily reduced. A panicle enters this low-field region un an open field line and becomes trapped in the field of one of the dipoles as the dipoles move apar. Subseyuently the perticle executes nounce orbits in the field of one of the dipoles. and is freed only hy an encounter with another moving dipole. Three-hody "recombination" events of this form are frequently observed in the vimulations. but they result in no net energy gain fior the particle. Sumewhat less common are events in which a particle is trapped between two or more dipoles. but these events are exceedingly important for particle acceleration because when these. traps are converging. very large energy gains cian be realized.

We accumulate histograms of the initial and final panicle energies. mensure the amount of bulk heating. the index of the power-law tuil (if any). the elficiency of hoosting (defined as the number of particles with tinal energies grenter than ten times the initial thermal energy divided by the total number of paricles). We also examine initial and final energies of the hoxosted particles. to characterize the population firnm which energized paricles are drawn. These statistics are summarized in Table I.

\begin{tabular}{|c|c|c|c|c|c|}
\hline \multirow[b]{2}{*}{ Geometry } & \multicolumn{4}{|c|}{ 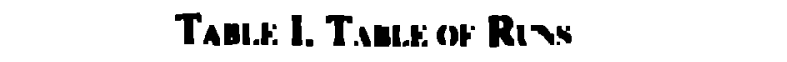 } & \multirow[b]{2}{*}{$\begin{array}{r}\text { Bulk } \\
\text { IIeatung }\end{array}$} \\
\hline & $\begin{array}{l}\text { Swarm } \\
\text { Speed }\end{array}$ & $\begin{array}{r}\text { Number } \\
\text { of } \\
\text { Panticles }\end{array}$ & $\begin{array}{r}\text { Iifficiency } \\
\text { of } \\
\text { Boxisting }\end{array}$ & $\begin{array}{l}\text { Index } \\
\text { of power } \\
\text { law tuil }\end{array}$ & \\
\hline sphencal & $0 .(10) 2$ & A7I & (1.29י & -1.76 & T.1. \\
\hline sphencul & $0.0 \times 16$ & (12474 & $0.31) 14$ & 1.22 & $1 .(1) 4$ \\
\hline sphencil & 0.02 & 74503 & $0.41 \%$ & $. \mid .5]$ & 1.14 \\
\hline sphericul & 0.04 & +59.5 .3 & $0.97 r_{i}$ & -1.27 & 1.3 .3 \\
\hline sphericul & 0.1 & 58744 & 1.187 & -1.15 & 1.80 \\
\hline sphenoul & 0.3 & +5025 & 8.707 & $-2.6 \mathrm{H}$ & 3.85 \\
\hline spherncul & 0.38 & 542.35 & 1.3..32., & -2.27 & 4.66 \\
\hline disk. 13/. & 0.02 & $\$ 1230$ & $0.1 y r_{7}$ & .1 .27 & 1.07 \\
\hline disk. Bx & 0.02 & $4(19.50$ & $0.21 r$ & -1.56 & 1.06 \\
\hline disk & 0.02 & +1074 & 0.237 & -1.09 & 1.09 \\
\hline uptunial swarm* & 0.02 & 10156.1 & 11.01517 & .2 .64 & $5 . n !$ \\
\hline
\end{tabular}

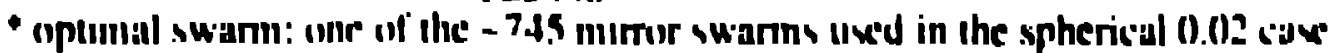

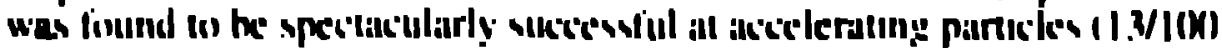

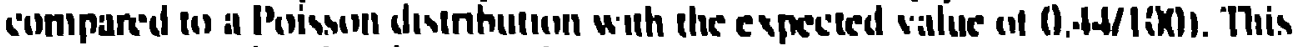
swarm u is isolated and run. Without reshufining the swirm. 


\section{Resi't.Ts}

When the swirm thermal speed is only a little less than the thermal speed of the particle population. as in Figure I. the ouiput spectrum looks very much like the initial one. just shifted to higher energy. The purricle population has imply been heated by its contact with the dipole swarm.

Figure 1. Initial and final energy histograms from a simulation in which the mirror swarm thermal velocity is comparable to the particle population thermil velocity. A total of 45.025 paricles were run in this simulation. The population is mostly buik heated. with very little production of a power-law tail to high energy.

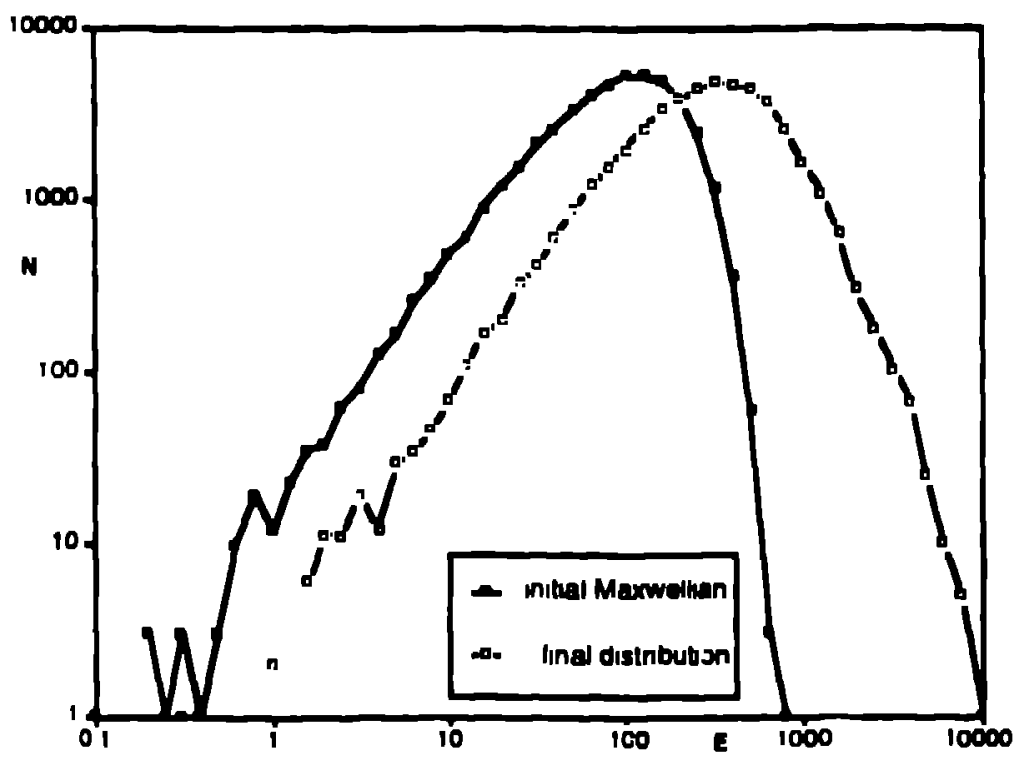

On the other hand. when the dipole swarm thermal speed is small compared tn the particle population thermal speed. as in Figures 2 and .3. there is mo bulk heating. but instead the production of a modest suprathermal tail. About as many particles are dropped into a low-energy tail also. As expected. the acielerution process is relatively inefficient. and both the boosting efficiency and the slope of ti.. power-law tiol depend on the relative slze of the swarm thermal speed. 
Figure 2. Initial and final energy histograms from a simulation in which the mirror swarm thermal velocity is $1 / 20$ times the particle population thermal velocity. A total of 74.503 paricles were run. and 327 of thcm were boosted into the suprathermal thil with final energies $>1000$. for an acceleration efficiency of 0.44\%. The high

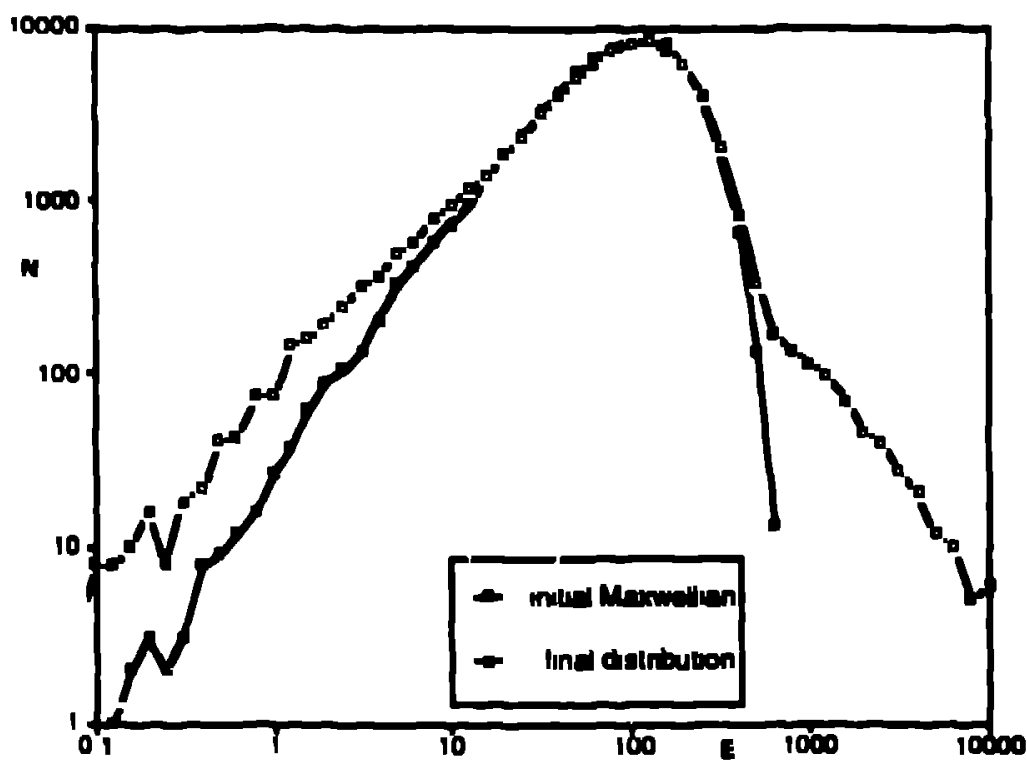
energy tail has a power-law index of -1.34 . There is no bulk heating of the population.

Figure 3. As Figure 2. but now the mirror swarm thermal veloxity is 1/200 times the particle populaion thermil velocity. A total of 44.729 particles were run. and 130 of them were horosited into the suprathermal tail with final energies > I000). for an accelera. tion efficiency of $0.29 \%$. The high eneryy tail has a

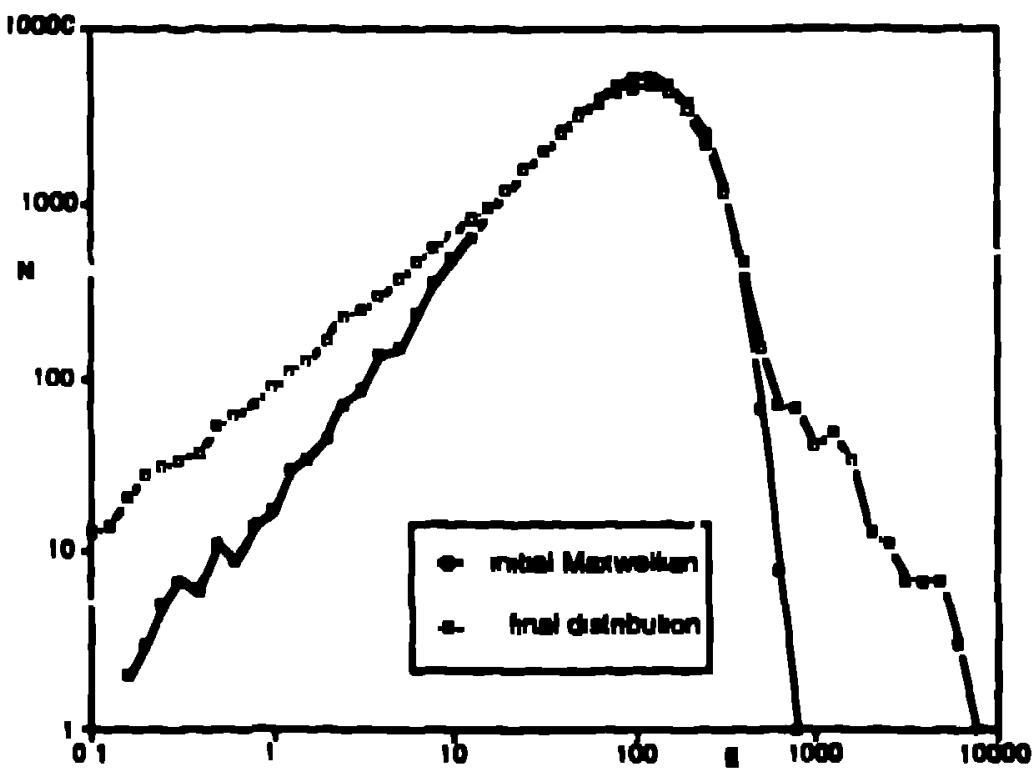
nower-law index of - 1.55. Again there is no hulk heating of the population.

An alualysis of those paricles in the suprathermal tail in all the runs of 'Table I yields the some what surprising result that nost of them originate at energies well helow the moss probable energy in the onginal distribution (see Figure d). In fact. hecuuse of the linise extent of the dipole swurm. parnicles that start out at higher energes lind to have lewer encoungers with the nimors hefore excaping, si have fewer opponunities lu gain energy. The paniclen starting out at low energies sulfer more eix'ounters and hence are more likely of he involved in a trapping event. Single 
dipole traps. as mentioned earlisr. do not result in energy gains. but converging multi-dipole traps can produce enomous energy gains with the result that a particle is xaltered far trom its original energy.

The trapping of a parucle in a converging mulu-dipole trap is a relatively improbable event. so those panicles that end up in the suprathermal tail are a very small fraction of the toral number of particles studied.

Figure 5. Comparison of initial and final energies for boosted particles from all runs. Boosted particles are defined as those particles with final energies greater than ten times the initial thermal energy. Also shown here is the shape of the inicial Maxwellian, scaled to the total number of boosted

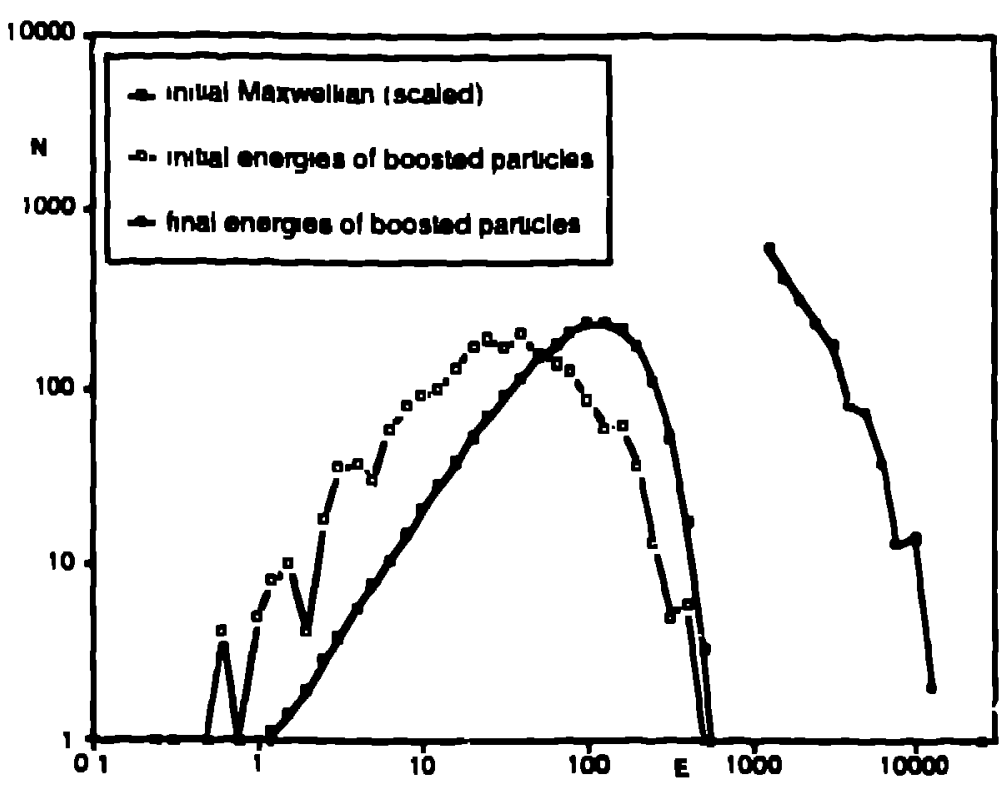
paricles. The boosted particles are found to originate in a distribution that is substantially cooler than the bulk of the population.

As mentioned before. the swarm ol dipoles is undergoing, as a whole. a thermal expension. (ne might expect that a typical particle in such a swarm wiould lose energy, since receding ninor veloxities predominate. Indeec many particles lose a substantial amount of energy. as evidenced by the low-energy tails in Figure:, 3 and 4. But also occurring is the production of a power-law high-energy tail.

Thermal expansions are extremely common in astrophysics. Stellar atmospheres and winds. ItII regions heated by hot young stars. supernova remnants, and galactic jess are but a few of the examples of such expansions. While the obvious result of a thermal expansion is an adiabatic cooling of the expanding gas, the acceleration of a few paricles to high energies may also oxcur.

\section{A ckNowl.fiximints}

This work was done under the auspices of the I!. S. Department of I:ncrgy's (Office of Basic linergy Sciences.

\section{Rtrine:vers}

I. M. A. Forman. R. Ramaty, and E: (j. Zweibel, in I'husics of the Sun. Cd. P. A. Sturroxk. T. L:. Holz.cr. I). M. Mihalas, and R. K. ('Irich; D), Reislei. Volume 2. pp 2.19-289 (1986).

2. M. A. Miller. N. (inessoum. and R. Ramaty. Astrophy.s. J. 361. 70) (194()).

3. (i. (iisler and D. I.emons. J. (ie(ophy.r. Res. 95. 1492.5 (1990). 